\title{
Analysis of Hysteresis Phenomenon as Observed from Voltammetric Data of Conducting Polymers. Part I
}

\author{
Tulio Matencio $^{*, a}$, Jean Michel Pernaut ${ }^{\dagger, a}$ and EricVieil ${ }^{b}$ \\ ${ }^{a}$ Departamento de Química, ICEx, Universidade Federal de Minas Gerais, CP 702, \\ 31270-901 Belo Horizonte - MG, Brazil \\ ${ }^{b}$ CEA, Département de Recherche Fondamentale sur la Matière Condensée, Laboratoire d'Electrochimie \\ Moléculaire, 38054 Grenoble Cedex 9, France
}

\begin{abstract}
Estudamos o fenômeno de histerese inerente aos processos redox dos polímeros condutores eletrônicos (PCE). As histereses foram separadas em duas classes: $i$ ) as histereses dinâmicas constituídas por componentes cinéticas e ôhmicas e ii) as histereses estacionárias ou termodinâmicas. Efetuamos uma análise matemática das histereses dinâmicas observadas nos voltamogramas cíclicos de dois polímeros: a polianilina (PANI) e o poli(3-metiltiofeno) (P3MT). Mostrou-se que no caso do P3MT a histerese é principalmente cinética devido à velocidade de uma das etapas do processo redox com também uma componente ôhmica relacionada à existência de uma impedância elétrica em serie com o sistema eletroquímico. No caso da PANI, observou-se que a histerese é independe da corrente, o que é característico de uma histerese termodinâmica.
\end{abstract}

The hysteresis phenomenon inherent to redox processes of Electronic Conducting Polymers (ECP) has been studied. Hysteresis was divided into two classes: i) dynamic hysteresis, containing a kinetic and an ohmic component and ii) stationary or thermodynamic hysteresis. A mathematical analysis was performed on the dynamic hysteresis observed in cyclic voltammograms of two polymers: polyaniline (PANI) and poly(3-methylthiophene) (P3MT). It has been shown that in the case of P3MT, the hysteresis is mainly kinetic due to the rate of one of the steps of the redox process. However, an ohmic component related to the existence of an electrical impedance in series with the electrochemical system is also present. As for PANI, hysteresis is current independent, which is characteristic of thermodynamic hysteresis.

Keywords: conducting polymers, hysteresis, cyclic voltammetry

\section{Introduction}

The hysteresis phenomenon occurring in redox processes of PCE has been evidenced by several experimental means, including electrochemical, ${ }^{1-5}$ spectroelectrochemical, ${ }^{1,6,7}$ and spectrophysical ${ }^{1,8-12}$ techniques. This type of hysteresis can be characterized through the charge-potential curves of the surface redox reaction, if no mass transfer kinetically limits the transformation. Under these conditions, it can be observed that the charge variation during the reverse cycle does not coincide with that observed during the forward sweep. In other words, the redox process is not reversible,

* e-mail:Tullio@dedalus.lcc.ufmg.br

$\dagger$ This paper is in memoriam of Jean Michel Pernaut (August 8, 1960 - August 25, 2002), who will always be remembered with gratitude, appreciation and kindness for his friendliness and wit by those who had the privilege of knowing him. i.e., not close enough to thermodynamic equilibrium. Some authors proposed explanations for this abnormal behavior of ECP redox mechanisms such as conformational change of polymeric chains, ${ }^{1-3}$ solvent and electrolyte movements, ${ }^{1,13-15}$ relaxation effects, ${ }^{1,16,17}$ phase transition ${ }^{1,7,18}$ or inter-site interactions, ${ }^{19}$ and, more recently, dimerizable redox sites. ${ }^{20}$

The aim of this work was to study the hysteresis phenomenon observed in charge-potential curves derived from cyclic voltammetry experiments performed on two ECPs, namely PANI and P3MT. The consequences of hysteresis are very important in the study of ECP redox behavior, since properties like the number of exchanged electrons, the redox potential and the electron transfer rate are not directly accessible without suitable data treatment. As mentioned before, several approaches have been developed with certain success to account for this non- 
ideal behavior, depending on the nature of the physical processes assumed to be important during the redox reaction. Here, a description of the different types of hysteresis, thermodynamic and kinetic are presented, and for the latter an original mathematical analysis is developed based on simple concepts and physical properties of conducting polymers such as electrical resistances. The models are then tested for both the polymers cited above and the results are discussed in terms of some physical properties.

Complementary results about hysteresis derived from relaxation potential experiments will be presented later.

\section{Experimental}

Commercially available aniline (R.P. Normapur) was distilled under primary vacuum. 3-methyl thiophene (Janssen, purity of 99\%) was distilled with sodium at $95^{\circ} \mathrm{C}$ under argon atmosphere. PANI films were electrochemically synthesized on a $\mathrm{Pt}$ substrate by cyclic voltammetry in $\mathrm{NH}_{4} \mathrm{~F}, 2.3 \mathrm{HF}$ solution containing $0.1 \mathrm{~mol} \mathrm{~L}^{-1}$ aniline. The scan rate was $50 \mathrm{mV} \mathrm{s}^{-1}$ and the potential window ranged from $-0.2 \mathrm{~V}$ to $0.65 \mathrm{~V}$ vs. $\mathrm{Cu} / \mathrm{CuF}_{2}$. P3MT film was electrochemically synthesized at a constant potential of $1.3 \mathrm{~V}$ vs. $\mathrm{Ag} / \mathrm{Ag}+$ in a propylene carbonate solution containing $0.01 \mathrm{~mol} \mathrm{~L}^{-1}$ (3-methyl) thiophene and $0.1 \mathrm{~mol} \mathrm{~L}^{-1} \mathrm{LiClO}_{4}$. All films studied had about the same thickness of approximately $1 \mu \mathrm{m}$, as calculated from the electroactive charge. ${ }^{21}$ Hysteresis studies were carried out from cyclic voltammetry experiments in $4 \mathrm{~mol} \mathrm{~L}^{-1} \mathrm{H}_{2} \mathrm{SO}_{4}$ aqueous solution for the PANI film and in propylene carbonate solution containing $0.1 \mathrm{~mol} \mathrm{~L}^{-1} \mathrm{LiClO}_{4}$ for the P3MT film.

\section{Results and Discussion}

Theoretical analysis

The analysis that follows is developed for a general redox system and then illustrated for a single charge transfer (faster or slower) occurring in a thin layer (in which diffusion/migration can be disregarded) and eventually submitted to non-ideal factors like electrical resistances.

\section{Dynamic hysteresis}

Kinetic hysteresis (vertical). The slowness of one of the steps of the redox mechanism, i.e. the kinetically determinant step, turns the redox behavior irreversible. This kind of hysteresis is quantitatively expressed by the decomposed charge, $Q$, which is observed at a given potential, into a reversible amount, $Q_{\text {rev }}$, independent of current, and a characteristic term of hysteresis, $H_{y}$, dependent on current.

Figures 1a and 1b, respectively, represent the theoretical variations of current and charge during cyclic voltammetric runs performed at different sweep rates for a quasi-reversible single charge transfer occurring in a thin layer.
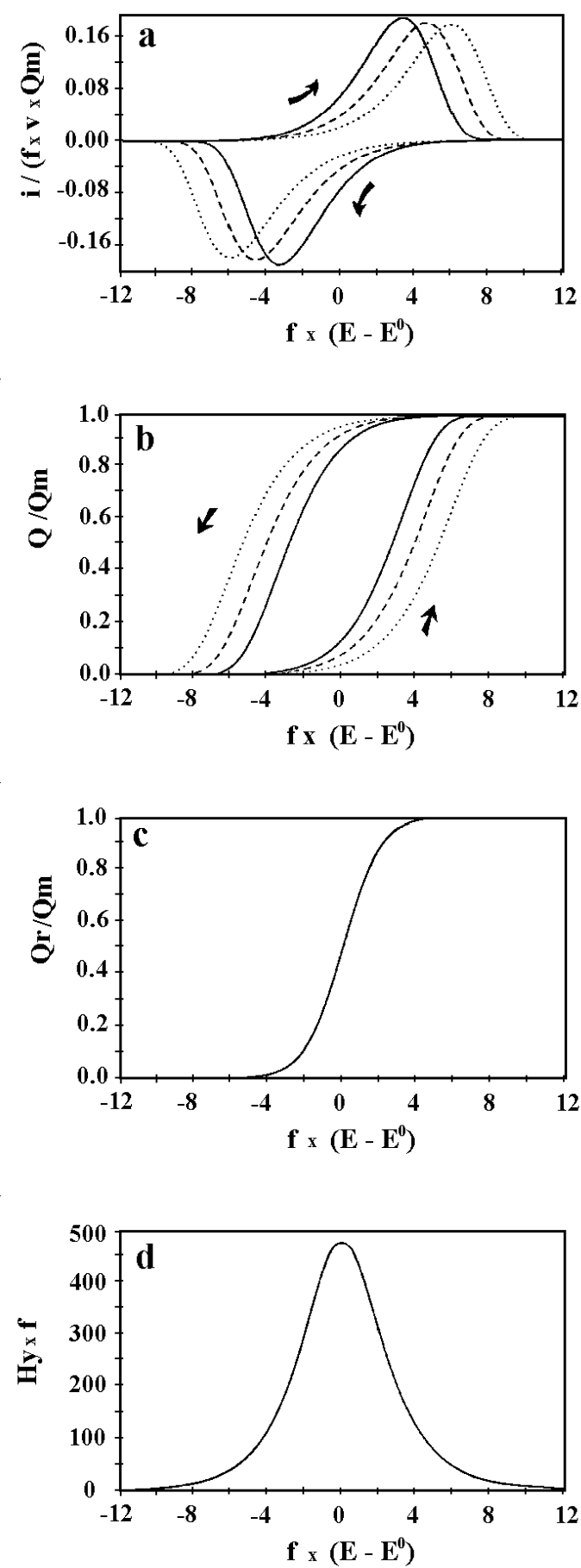

Figure 1. a) Cyclic voltammograms and b) corresponding charge for a quasi-reversible transfer. Scan rates are (-) 10, (---) 20 and (..) $40 \mathrm{mV} \mathrm{s}^{-1}$; c) Reversible charge; d) Kinetic hysteresis. 
The analysis consists in considering a vertical hysteresis. For a given potential the system can take two different states. Then, assuming that for a given potential the hysteresis is the same for the forward and backward sweep, one can write:

$Q_{A}(E)=Q_{\text {rev }}(E)-H_{y}(E) \cdot i_{A}(E)$

$Q_{B}(E)=Q_{r e v}(E)-H_{y}(E) \cdot i_{B}(E)$

where $i_{A}(\mathrm{E}), i_{B}(\mathrm{E})$ represent the anodic and cathodic currents, respectively, and $Q_{A}(\mathrm{E}), Q_{B}(\mathrm{E})$, the anodic and cathodic charges, respectively.

For each potential value, the reversible charge is expressed as:

$Q_{r e v}(E)=\frac{i_{B}(E) \cdot Q_{A}(E)-i_{A}(E) \cdot Q_{B}(E)}{i_{B}(E)-i_{A}(E)}$

and the hysteresis component $\left(\mathrm{s}^{-1}\right)$, which quantifies the deviation from ideality, can be written as:

$H_{y}(E)=\frac{Q_{A}(E)-Q_{B}(E)}{i_{B}(E)-i_{A}(E)}$

It is noteworthy that for any redox thin layer system submitted to a cyclic potential sweep, expressions (3) and (4) allow deducing the reversible charge and vertical hysteresis evolutions from current- and charge-potential curves.

As an illustration, the analysis of the kinetic hysteresis was carried out for a quasi-reversible single charge transfer occurring in a thin layer. Figures $1 \mathrm{c}$ and $1 \mathrm{~d}$ show the reversible charge and $H_{y}$ hysteresis component, respectively, obtained from the kinetic hysteresis analysis of Figures 1a and 1b.

It is possible to check the validity of our analysis, since in the case of a single charge transfer occurring in a thin layer, the relation between charge and potential is:

$\frac{Q}{Q^{m}}=\frac{1-\frac{i}{i_{0}^{L}} \exp \left(-\alpha_{A} X_{f}\right)}{1+\exp \left(-X_{f}\right)}$

with $X_{f}=-n f\left(E_{f}-E^{0}\right)$ where $\mathrm{n}$ is the number of electrons (n $>0$ for reduction and $\mathrm{n}<0$ for oxidation), $E_{f}$ is the potential experienced by the faradic process, $E^{0}$ is the normal redox potential, and $f=F / R T$. $Q$ is the faradic charge, $Q^{m}$ is the maximum faradic charge $\left(Q=Q^{m}\right.$ when $\left.E_{f}>E^{0}\right)$ and $i_{0}^{L}=-n F A C_{T} K^{0}$. From relations (2), (3) and (5):

$Q_{\text {rev }}=\frac{Q^{m}}{1+\exp \left(-X_{f}\right)}$ and

$H_{y}=\frac{Q^{m} \exp \left(-\alpha_{A} X_{f}\right)}{i_{0}^{L}\left(1+\exp \left(-X_{f}\right)\right)}$

It can be noticed that the faster the charge transfer (large $\left.i_{0}^{L}\right)$, the weaker the $H_{y}$ component, and as a result, the more reversible the system becomes. In this way, $H_{y}$ is a measure of the global reversibility of the redox reaction regardless of the nature of the charge transfer.

Ohmic hysteresis (horizontal): The presence of an impedance element, $H_{x}$, in series with the electrochemical system under study causes the non-superposition of forward and backward currents due to the current dependence of the apparent potential of the system.

Figures $2 \mathrm{a}$ and $2 \mathrm{~b}$ illustrate the theoretical current and charge changes, respectively, for a single charge transfer occurring in a thin layer without interactions between redox sites and with the presence of a series resistance during cyclic voltammetric runs at various scan rates.

The analysis consists of considering an horizontal hysteresis, which means that the system can be in the same state for two different potentials. Then, assuming that for the same state of the material (film or polymer thin layer) electrochemically characterized by its electrical charge, the hysteresis is the same for the forward and backward sweeps, one can write:

$E_{A}(Q)=E_{\text {rev }}(Q)+H_{x}(Q) \cdot i_{A}(E)$
$E_{B}(Q)=E_{\text {rev }}(Q)+H_{x}(Q) \cdot i_{B}(E)$

where $E_{A}(\mathrm{Q}), E_{B}(\mathrm{Q})$ represent the forward and backward potentials, i.e. the potentials associated with the anodic and cathodic charges, and $i_{A}(\mathrm{E}), i_{B}(\mathrm{E})$ represent the anodic and cathodic currents, respectively.

For one state of the system, the reversible potential, which is the potential without ohmic hysteresis, can be expressed as:

$E_{\text {rev }}(Q)=\frac{i_{B}(E) \cdot E_{A}(Q)-i_{A}(E) E_{B}(Q)}{i_{B}(E)-i_{A}(E)}$

and the hysteresis (Ohms) component is:

$H_{x}(Q)=\frac{E_{A}(Q)-E_{B}(Q)}{i_{B}(E)-i_{A}(E)}$

$H_{x}$ component corresponds here to a series resistance, presumably identical for forward and backward scans, 

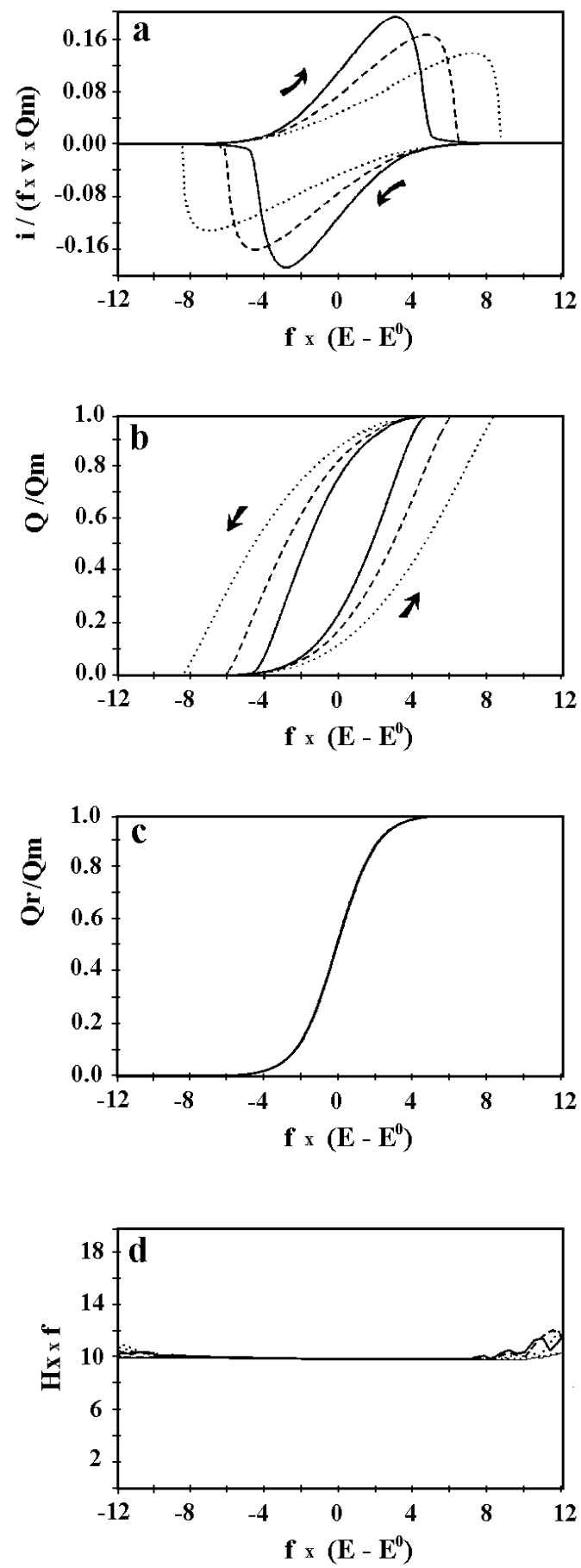

Figure 2. a) Cyclic voltammograms and b) corresponding charge for a reversible charge transfer with a series resistance. Scan rates are (-) 10, (--) 20 and (..) $40 \mathrm{mV} \mathrm{s}^{-1}$; c) Reversible potential; d) Ohmic hysteresis.

because the state of the material, characterized by its electrochemical charge, is the same for both scans.

It is noteworthy that for any redox thin layer system submitted to a cyclic potential sweep, expressions (10) and (11) allow deducing the reversible potential and horizontal hysteresis evolutions from experimental currentand charge-potential curves.

As an illustration, the analysis of an ohmic hysteresis was carried out for a reversible single charge transfer occurring in a thin layer along with a series resistance. Figure 2c shows the $E_{\text {rev }}$ potential experienced by the nonresistive part, and Figure $2 \mathrm{~d}$ shows the variation of ohmic hysteresis obtained from ohmic hysteresis analysis of Figures $2 a$ and $2 b$. Here, the validity of the analysis can be checked by: $i$ ) Nernst evolution of $E_{\text {rev }}$ potential, which fits well a reversible charge transfer, $i i)$ identification of the $H_{x}$ component with the series resistance.

This series resistance can be understood as the ohmic non-compensated resistance, that can include, depending on the experimental conditions, the electrical resistance of the electrolyte and of the thin layer, which is variable in the case of an ECP.

Oblique hysteresis: This type of hysteresis occurs when ohmic and kinetic hystereses co-exist. The hysteresis analysis was carried out from cyclic voltammograms recorded at several sweep rates. The expressions of the ohmic and kinetic components are:

$H_{x}(Q)=\frac{E_{1}(Q)-E_{2}(Q)}{i_{1}(E)-i_{2}(E)}$

and

$H_{y}(E)=\frac{Q_{1}(E)-Q_{2}(E)}{i_{2}(E)-i_{1}(E)}$

where $Q_{1}(E), Q_{2}(E), i_{1}(E), i_{2}(E), E_{I}(Q), E_{2}(Q)$ are charges, currents and potentials for sweep rates $\mathrm{v}_{1}$ and $\mathrm{v}_{2}$, respectively. In order to explain the underlying principle used to determine the $Q_{1}(E), Q_{2}(E), i_{1}(E), i_{2}(E), E_{l}(Q), E_{2}(Q)$ parameters, a quasi-reversible charge transfer was considered in series with a resistance. In this case, the equation to be solved corresponds to equation (5). The illustration of such a process is shown in Figures $3 \mathrm{a}$ and $3 \mathrm{~b}$ through charge and current changes obtained for two different sweep rates.

The treatment used here is based on the principle that for one given abscissa $E_{\text {rev }}$, there are several lines intersecting the two charge cycles. For each of these line, we obtain the intersection points $\mathrm{a}$ and $\mathrm{b}$ with the charge diagram at sweep rate $\mathrm{v}_{1}$ and points $\mathrm{c}$ and $\mathrm{d}$ at sweep rate $\mathrm{v}_{2}$ (Figure 3a). These intersection points correspond to points a', b', c' and d', respectively, on the current curves (Figure $3 b)$. The rotation of $a, b, c, d$ straight lines between horizontal and vertical limits is performed automatically 

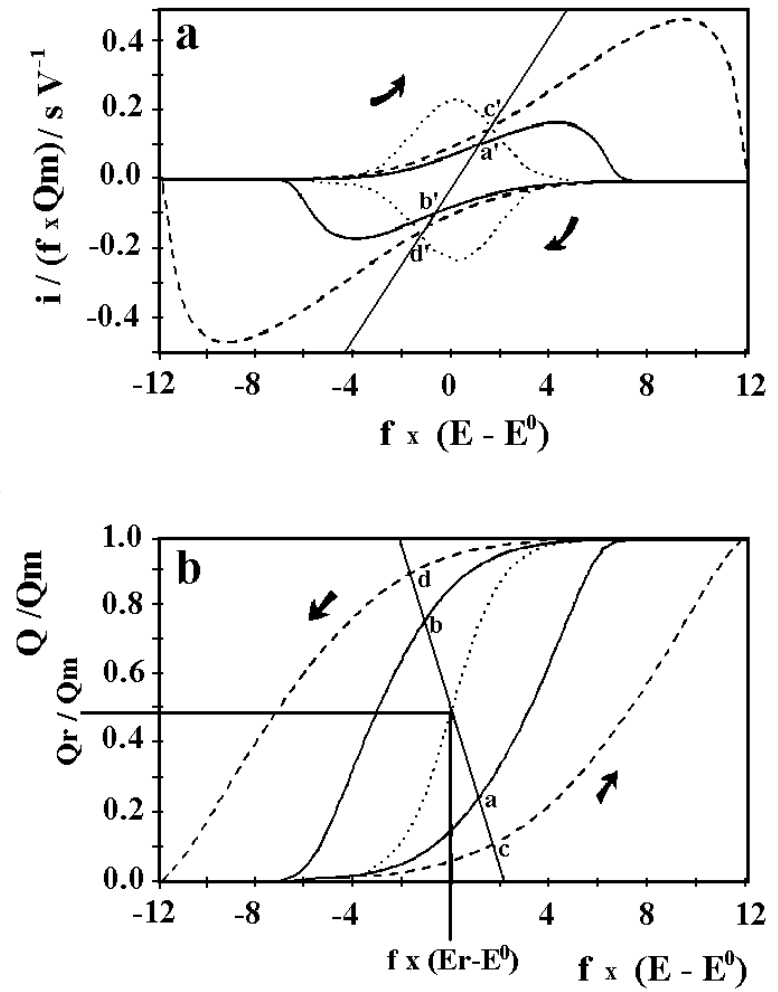

Figure 3. a) Theoretical cyclic voltammograms and b) corresponding charge for a quasi-reversible charge transfer with a series resistance. Scan rates are (-) $10,(--) 40 \mathrm{mV} \mathrm{s}^{-1}$. (..) Calculated reversible charge and current.

using a pre-defined increment until the corresponding a', b', c' and d' also form a straight line. Once this is achieved, it means that points $a, b, c$ and $d$ are characteristic of the kinetic hysteresis. The linearity of the a', b', c' and d' segment is checked by a linear regression algorithm based on a least squares determination. Finally, relations (12) and (13) can be obtained using relations (3), (4), (10), and (11).

\section{Stationary hysteresis}

In contrast to the other classes of hysteresis dependent on current, and consequently on sweep rate, here hysteresis is independent of current. Many reports can be found in electrochemical literature, in agreement with the situations in which interactions between species ${ }^{22,23}$ occur. Physically, in the case of ECP, these types of interactions could be electrostatic or mechanical in nature due the insertion of counter-ion. ${ }^{24}$ For example, Felberg and Rubistein ${ }^{25}$ presented some physical and electrochemical cases of this kind of hysteresis that most often are followed by a kinetic or ohmic limitation with successive rearrangements due to deviation from stability. This category of hysteresis can be expressed with the following linear relations:

$$
E_{A}(Q)=E_{\text {rev }}(Q)+H_{Z A}(Q) \cdot Q_{A}(E)
$$

and

$$
E_{B}(Q)=E_{\text {rev }}(Q)+H_{Z B}(Q) \cdot Q_{B}(E)
$$

where $E_{\text {ver }}(Q)$ represents the potential component without hysteresis and $H_{Z A}(Q), H_{Z B}(Q)$ the factors for the hysteresis components. In opposition to the kinetic and ohmic components previously described, the hysteresis terms included in equations (14) and (15) are generally different from forward and backward scans. Moreover, generally it is not possible to express $E_{r e v}, H_{Z A}$ and $H_{Z B}$ independently one from another, and their mathematical analysis would require a complex development which would be beyond the scope of this study.

Figure 4 represents an example of stationary hysteresis with charge variation during an electrochemical process with an irreversible charge transfer, and linear interaction between the various redox species using a known model. ${ }^{19}$

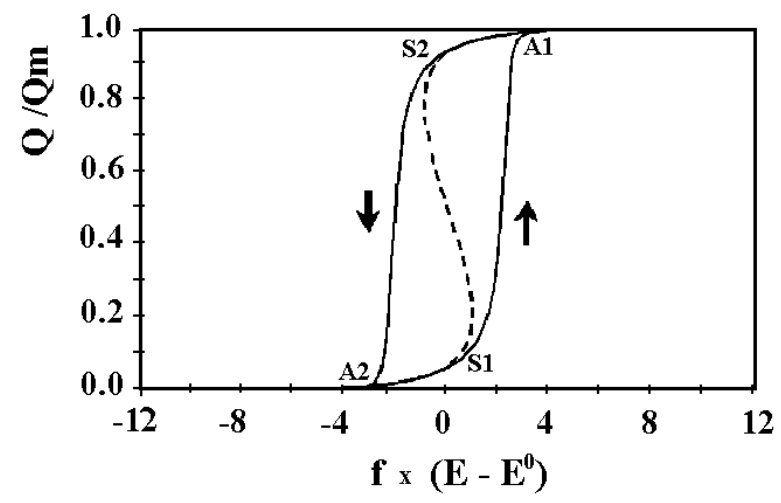

Figure 4. Illustration of hysteresis in a charge curve. This corresponds to an irreversible charge transfer with linear interactions (electrostatic or mechanical type) between the redox species. (-) real charge (---) reversible charge.

The interpretation of this type of hysteresis, which depends mainly on the system state and not on the scan rate, can be stated as follows: during the forward sweep the system does not exhibit any hysteresis up to the $Q_{S 1}$ charge value, but above this value, the inter-species forces facilitate the oxidation reaction by lowering the energy necessary to complete the redox process. The dotted curve between $\mathrm{S} 1$ and $\mathrm{S} 2$ represents the thermodynamic equilibrium of the system, which is achieved with potentials lower than $\mathrm{E}_{\mathrm{S} 1}$. However, since the potential is imposed, the system can evolve very quickly to the next equilibrium state available for a potential higher than $\mathrm{E}_{\mathrm{S} 1}$, i.e., to reach point $\mathrm{A} 1$, and the charge variation occurs by a jump until it reaches its maximum. 
During the reverse sweep, the system does not show any hysteresis up to the $Q_{S 2}$ charge value. Above this value, the phenomenon previously described occurs, making reduction more difficult. Furthermore, the constant decrease in the potential results in a sharp charge fall down to its minimum value. In our example, for the same potential, the system can be in three different states, but it is possible to consider cases with a greater number of metastable states. Physically, these states could be associated, for example, with different local structures of the polymer. Moreover, this implies that the transitions between points $\mathrm{S} 1$ and $\mathrm{A} 1$, and points S2 and A2 cannot be infinitely fast. Indeed, there is an inevitable kinetic limitation which absorbs the sudden dissipation of energy. This is represented here by a limitation of the charge transfer, for which an intrinsic finite $\mathrm{K}^{0}$ constant rate has to be considered.

\section{Application to ECP}

Figures $5 \mathrm{a}$ and $5 \mathrm{~b}$ represent the charge evolution at various scan rates during the redox process of P3MT and PANI, respectively.
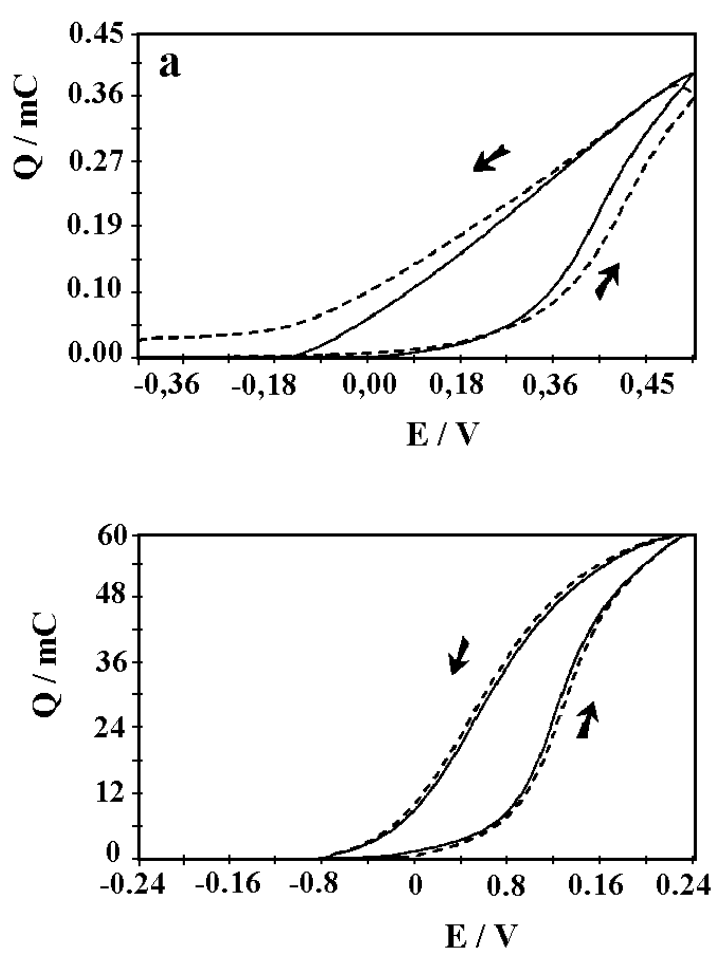

Figure 5. Charge curves at scan rates of (-) 20 and (---) $50 \mathrm{mV} \mathrm{s}^{-1}$ during redox process of a) P3MT and b) PANI.

In the case of P3MT, hysteresis varied according to the scan rate, whereas in the case of PANI, the charge evolution was independent of the scan rate. This led us to conclude that dynamic hysteresis played a more significant role in the case of P3MT than in the case of PANI. Using the procedure developed for the study of oblique hysteresis, the charge curve was analyzed, and the reversible charge (Figure 6a), and the resulting kinetic and ohmic hysteresis components (Figure 6b) were determined.
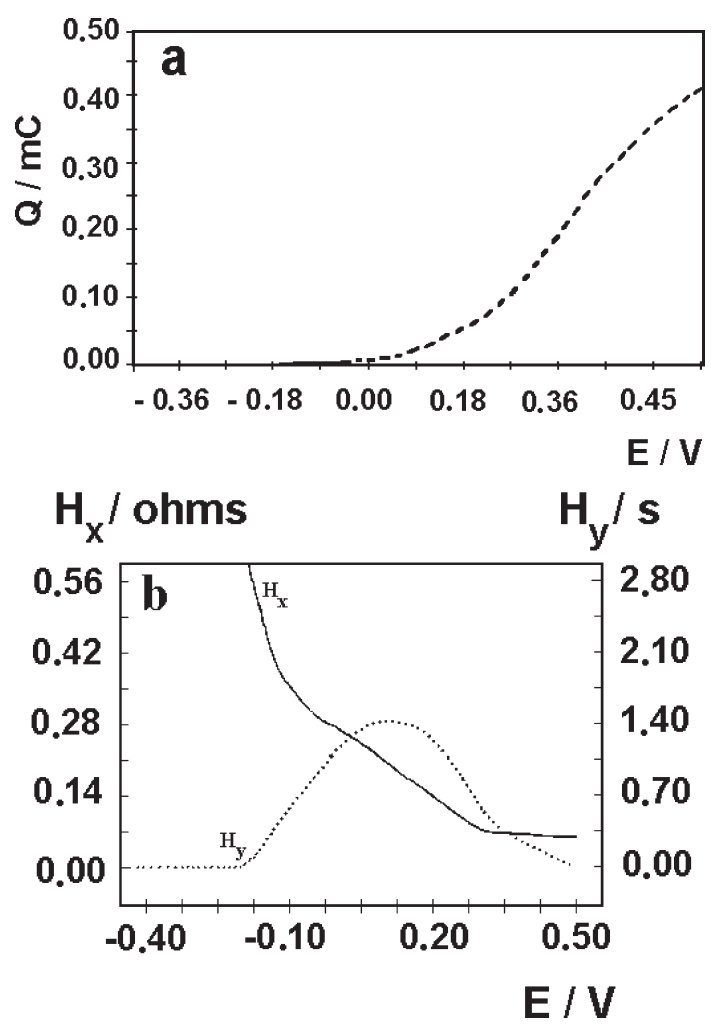

Figure 6. a) Reversible charge and b) ohmic hysteresis components determined by oblique hysteresis calculation.

A quick examination of the values made evident that in the latter case the contribution of the kinetic hysteresis is much larger than that of the ohmic hysteresis. The evolution of this kinetic hysteresis is the same as that in the case of a quasi-reversible charge transfer occurring in a thin layer. ${ }^{26}$ The variation of ohmic hysteresis is in agreement with the fact that ECPs are insulators in their reduced states and increase their conductivity during the oxidation process up to a maximum value in their fully oxidized state.

In the case of PANI, hysteresis remained constant, independent of the scan rate, thus, the analysis of the oblique hysteresis contribution was not possible. In order to get more kinetic and thermodynamic data on the electrochemical behavior of PANI, a potential relaxation study has been carried out. This study will be published later. 


\section{Conclusion}

The study of the hysteresis phenomenon associated with the redox process of electronic conducting polymer led us to distinguish two types of hysteresis: dynamic and stationary. Dynamic hysteresis may have a kinetic origin, and in this case, it is related to the rate of one of the steps of the redox mechanism, or it may display an ohmic origin, and therefore be caused by the presence of an electrical resistance in series with the system. Stationary hysteresis, in opposition to the other types of hysteresis, does not depend on current. It can be found in all systems that present several metastable states or significant interactions between electrochemical sites. The development of a mathematical treatment of dynamic analysis of hysteresis allowed us to study the hysteresis phenomenon present in the redox process of two electroactive polymers: PANI and P3MT. It has been shown that in the case of PMT, hysteresis has mainly kinetic and ohmic origins, whereas in the case of PANI, the hysteresis observed is independent of scan rate and current, and seems more likely to have a thermodynamic origin. Additional information on the stationary hysteresis of the redox process of PANI has been obtained from potential relaxation experiments and will be presented elsewhere.

\section{Acknowledgements}

We are grateful to the Brazilian Research Agencies, Fapemig and CNPq, for financial support.

\section{References}

1. Inzelt, G.; Pineri, M.; Schultze, J.W.; Vorotyntsev, M. A.; Electrochim. Acta 2000, 45, 2403.

2. Grande, H.; Otero, T.F.; J. Phys.Chem. B 1998, 102, 7535.
3. Grande, H.; Otero, T.F.; Eletrochim. Acta 1999, 44, 1893.

4. Vorotyntsev, M.A.; Vieil, E.; Heinze, J.; J. Electroanal. Chem. 1998, 450, 121.

5. Meerholz, K.; Heinze, J.; Electrochim. Acta 1996, 41, 1839.

6. Visy, C. ; Kankare, J.; J. Electroanal. Chem. 1998, 442, 175.

7. Poks, P.; Grzeszczuk, M.; Synth. Met. 1997, 89, 237.

8. Kang, E.T.; Neoh, K.G.; Tan, K.L.; Polymer 1996, 37, 925.

9. Meerholz, K.; Swiatkiewicz, J.; Prasad, P. N.; J. Phys. Chem. 1995, 199, 7715 .

10. Odin, C.; Netchtschein, M.; Synth. Met. 1991, 43, 2943.

11. Matencio, T.; Peres, R. C. D.; Torresi, R.; Torresi, S.; Paoli, M.A.; Synth. Met. 1995, 72, 59.

12. Paik, W.;Yeo, I.H.; Suh, H.; Kin, Y.; Song, E.; Electrochim. Acta 2000, 45, 3833.

13. Dinh, H.N.; Birss, V.I. ; J. Eletroanal. Chem. 1998, 443, 63.

14. Yang, H.; Kwak, J.; J. Phys. Chem. B 1997, 101, 774.

15. Baudouin, J. L.; Chao, F.; Costa, M.; J. Chim. Phys. 1989, 86, 181.

16. Vorotyntsev, M.A.; Vieil, E.; Heinze, J.; J. Electroanal. Chem. 1996, 41, 1913.

17. Inzelt, G.; Electrochim. Acta 2000, 45, 2403.

18. Vorotyntsev, M. A.; Badiali, J.P.; Eletrochim. Acta 1994, 39, 289.

19. Pernaut, J.M.; Genies,E.M.; Nechtschein, M.; Vieil, E.; Genoud, F.; Devreux, F.; Synth. Met. 1986, 15,

20. Vorotyntsev, M.A.; Heinze, J.; Electrochim. Acta 2001, 46, 3309.

21. Pernaut, J.M.; Genies, E.M.; Synth. Met. 1984, 10, 117.

22. Laviron, E.; J. Electroanal. Chem. 1979, 100, 263.

23. Sadkowski, A. ; J. Electroanal. Chem. 1979, 105, 1.

24. Marque P.; Roncali J.; J. Phys. Chem. 1990, 94, 8614.

25. Felberg, S. W.; Rubistein, I.; J. Electroanal. Chem. 1988, $240,1$.

26. Vieil, E.; J. Electroanal. Chem. 1991, 297, 61.

Received: September 9, 2001

Published on the web: November 29, 2002 